\title{
Nm-sized cryogenic hydrogen clusters for a laser-driven proton source
}

Cite as: Rev. Sci. Instrum. 90, 043301 (2019); https://doi.org/10.1063/1.5080011

Submitted: 05 November 2018 . Accepted: 20 March 2019 . Published Online: 15 April 2019

S. Grieser, B. Aurand, E. Aktan, D. Bonaventura, M. Büscher (D, M. Cerchez, I. Engin, L. Leßmann, C. Mannweiler, R. Prasad, O. Willi, and A. Khoukaz

\section{ARTICLES YOU MAY BE INTERESTED IN}

Magnetic field stabilization system for atomic physics experiments

Review of Scientific Instruments 90, 044702 (2019); https://doi.org/10.1063/1.5080093

Soft X-ray nanoscale imaging using a sub-pixel resolution charge coupled device (CCD) camera

Review of Scientific Instruments 90, 04311 (2019); https://doi.org/10.1063/1.5053593

Design optimization of a fast-neutron detector with scintillating fibers for triton burnup experiments at fusion experimental devices

Review of Scientific Instruments 90, 043503 (2019); https://doi.org/10.1063/1.5074131

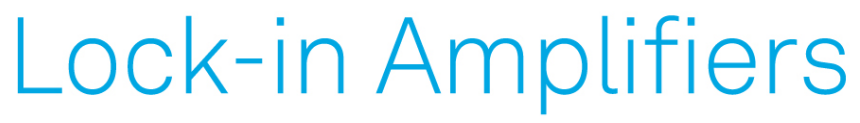

... and more, from DC to $600 \mathrm{MHz}$ Watch 


\title{
Nm-sized cryogenic hydrogen clusters for a laser-driven proton source
}

\author{
Cite as: Rev. Sci. Instrum. 90, 043301 (2019); doi: 10.1063/1.5080011 \\ Submitted: 5 November 2018 • Accepted: 20 March 2019 • \\ Published Online: 15 April 2019
}

\author{
S. Grieser, ${ }^{1, a)}$ B. Aurand, ${ }^{2, b)}$ E. Aktan, ${ }^{2}$ D. Bonaventura, ${ }^{1}$ M. Büscher, ${ }^{3}$ (D) M. Cerchez, ${ }^{2}$ I. Engin, ${ }^{3}$ L. Leßmann, \\ C. Mannweiler, ${ }^{\top}$ R. Prasad, ${ }^{2}$ O. Willi, ${ }^{2}$ and A. Khoukaz
}

\begin{abstract}
AFFILIATIONS
${ }^{1}$ Institut für Kernphysik, Westfälische Wilhelms-Universität Münster, 48149 Münster, Germany

${ }^{2}$ Institut für Laser- und Plasmaphysik, Heinrich-Heine-Universität, 40225 Düsseldorf, Germany

${ }^{3}$ Peter Grünberg Institut, PGI-6, Forschungszentrum Jülich, 52428 Jülich, Germany
\end{abstract}

a) Electronic mail: S_grie06@wwu.de

${ }^{\text {b) }}$ Electronic mail: bastian.aurand@hhu.de

\begin{abstract}
A cryogenic hydrogen cluster-jet target is described which has been used for laser-plasma interaction studies. Major advantages of the clusterjet are, on the one hand, the compatibility to pulsed high repetition lasers as the target is operated continuously and, on the other hand, the absence of target debris. The cluster-jet target was characterized using the Mie-scattering technique allowing to determine the cluster size and to compare the measurements with an empirical formula. In addition, an estimation of the cluster beam density was performed. The system was implemented at the high power laser system ARCTURUS, and the measurements show the acceleration of protons after irradiation of the cluster target by high intensity laser pulses with a repetition rate of $5 \mathrm{~Hz}$.
\end{abstract}

Published under license by AIP Publishing. https://doi.org/10.1063/1.5080011

\section{INTRODUCTION}

In the field of laser-induced ion acceleration, stability and applicability have tremendously increased during the last two decades. Starting from the process of target-normal-sheath-acceleration (TNSA), ${ }^{1,2}$ a variety of different acceleration schemes have recently been proposed including radiation-pressure-acceleration (RPA), ${ }^{3-5}$ collisionless-shock-acceleration (CSA), ${ }^{6,7}$ and break-outafterburner (BOA). 8,9 These different processes allow the improvement of the parameters of the laser-driven proton beams such as flux, energy distribution, and collimation. Generally, flat foil targets are used but also more sophisticated targets have recently been proposed. ${ }^{10,11}$ A detailed review of the recent progress achieved in the area of laser-ion acceleration can be found in the publications by Macchi. ${ }^{12,13}$

Two major disadvantages of most targets are the fact that they have to be replaced after every laser shot. In addition, a large part of the incident laser energy is lost by the escape of energetic electrons transversely to the laser propagation direction. ${ }^{14-16}$ Ideas to overcome the first problem are, for example, the use of gaseous targets. ${ }^{17-19}$ The drawback is the low mass density resulting in a low particle flux. Other ideas are the use of liquids or solids, dispersed in a jet-like structure. ${ }^{20,21}$ This guarantees unlimited target material at liquid or solid densities but comes with the need for a precise laser alignment onto the often very small objects. The second problem, the energy loss by the escaping electrons, could be overcome by the so-called mass-limited targets. In this case, however, the target with the very fine support structure or the fully levitated target ${ }^{22}$ has to be replaced after every laser shot.

By contrast, a cluster-jet target overcomes most of the difficulties mentioned above. The cluster source delivers, on the one hand, mass-limited targets in a continuous beam. On the other hand, the cluster density is high enough to have a sufficient density in the focal volume during the interaction. The clusters can be produced from a liquid $^{23}$ or a cryogenically cooled gas. ${ }^{24,25}$ In addition, a major benefit of a cluster source is the use of hydrogen gas at high purity allowing only protons to be accelerated. By contrast, most other targets contain hydrocarbons or oxygen resulting in the acceleration of heavier ions.

Recently, the use of micrometer-sized clusters of hydrogen for proton acceleration was demonstrated by Jinno et al. ${ }^{26}$ Here, we report on the development of a cluster target which can be used 
at a high repetition rate. The jet was characterized including the cluster size using the technique of Mie-scattering. The performance of the target by irradiation with a laser beam at an intensity of $I=10^{20} \mathrm{~W} / \mathrm{cm}^{2}$ was successfully tested, and protons in the kiloelectron volt range were observed.

\section{THE CLUSTER-JET TARGET}

The cluster-jet target MCT1S was designed and built at the University of Münster for nuclear physics studies. Detailed investigations were performed on the production of cluster-jet beams. In the first set of measurements, a hydrogen cluster beam was defined by a set of skimmers and studied up to $2 \mathrm{~m}$ behind the target beam generator. $^{27}$ This cluster-jet target was rebuilt and optimized for laser plasma interaction studies allowing the laser beam to be focused very close to the target generator (see Fig. 1) with an average target density of $>10^{16}$ atoms $/ \mathrm{cm}^{3}{ }^{28,29}$ Depending on the experimental setup, the cluster-jet target offers the possibility to cool gases to temperatures of roughly $T=20 \mathrm{~K}$. For the experiments presented here, the target was modified to allow for interaction studies $28 \mathrm{~mm}$ behind the nozzle exit (see Fig. 1) which increased the lowest temperature to roughly $T=57 \mathrm{~K}$. The (pre-cooled) gas is directed with pressures of, e.g., $p=16$ bar through a fine Laval nozzle with a typical diameter between $28 \mu \mathrm{m}$ and $100 \mu \mathrm{m}$. With a starting temperature well below the inversion temperature and due to the specific convergent-divergent shape of the nozzle, the gas is further cooled by adiabatic cooling during passing the nozzle and the formation of clusters develops. The cluster beam consists of individual solid clusters with a local electron/proton density of roughly $5 \times 10^{22} \mathrm{~cm}^{-3}$.

The Laval nozzle is one of the main components of a clusterjet target, and its complex inner geometry is essential for the cluster production process. The convergent short $90^{\circ}$ inlet cone merges after the narrowest point of the nozzle into a $17 \mathrm{~mm}$ long divergent outlet zone (cf. Fig. 1). The nozzles used here originate from production lines developed by CERN (Conseil européen pour la recherche

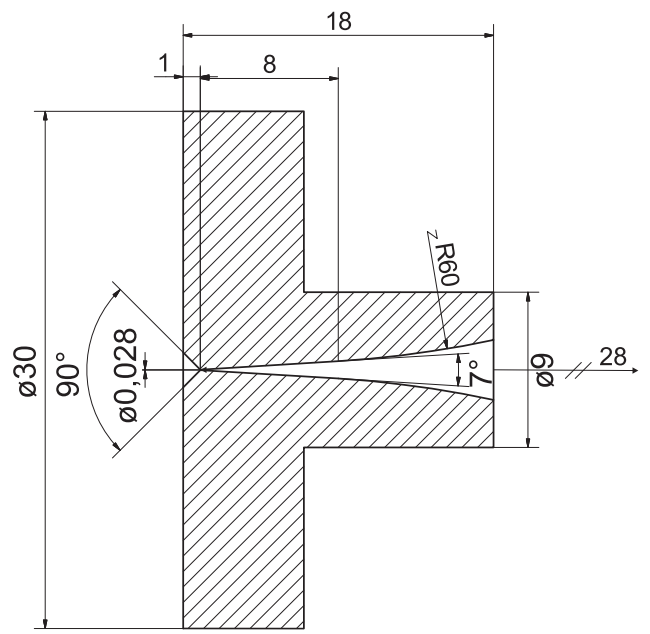

FIG. 1. Sketch of the Laval nozzle used in experiments. For hydrogen, an inner diameter of $\emptyset_{\mathrm{H}_{2}}=28 \mu \mathrm{m}$ was used. The dimensions are given in units of millimeter.
TABLE I. Target components and their specification.

\begin{tabular}{lc}
\hline \hline Target components & Specification \\
\hline Cold head & Leybold RGD 1245 \\
Compressor & Leybold Coolpak 6000H \\
Pressure controller & SLA 5810 (Brooks) \\
Flow meter & SLA 5860 (Brooks) \\
Hydrogen purifier & HP-50 (Johnson Matthey) \\
Baratron & 722 (MKS) \\
Temperature control & Lakeshore 206/336 \\
& PSP-603 (GwInstek) \\
Temperature diodes & 1. Stage: DT 670 (Lakeshore) \\
& 2. Stage: DT 670 (Lakeshore) \\
Heating cartridges & 1. Stage: 50 W (Lakeshore) \\
& 2. Stage: 100 W (Lakeshore) \\
\hline \hline
\end{tabular}

nucléaire). An improved production process was developed at the University of Münster to ensure the availability and the diversity of Laval nozzles for different gases and stagnation conditions.

The cluster-jet target can be used with different gases. Depending on the target material, the nozzle geometry has to be adapted in order to provide appropriate target thicknesses and gas flows through the nozzle for the different gases. Thereby, the general geometry of the nozzle was the same and only the narrowest inner diameter has to be varied. In case of hydrogen, the nozzle had an inner diameter of $\emptyset_{\mathrm{H}_{2}}=28 \mu \mathrm{m}$.

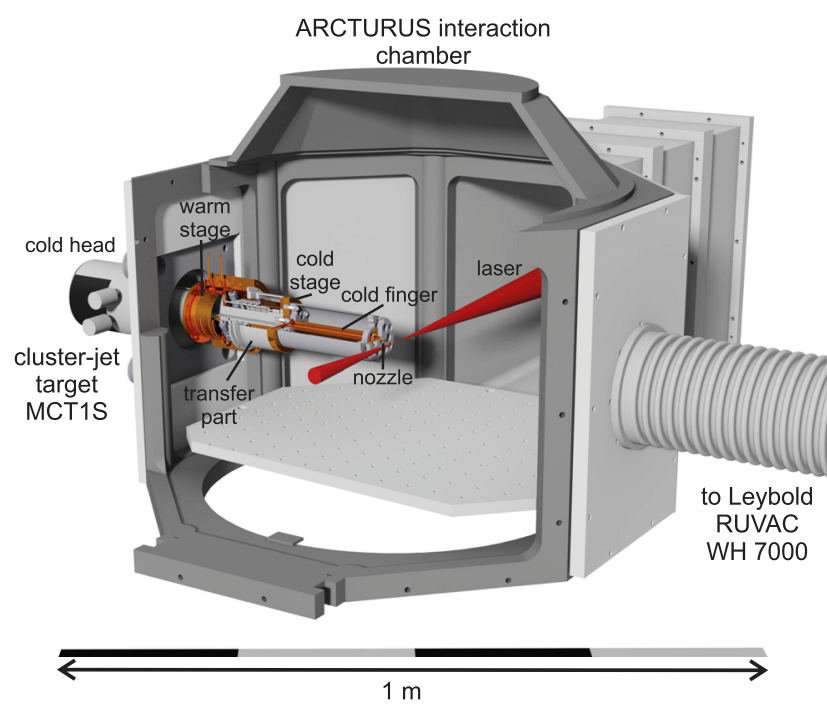

FIG. 2. The cluster-jet target MCT1S (left) installed at the interaction chamber of the ARCTURUS laser system in Düsseldorf. The cluster beam direction is from the left to right, and the interaction with the laser (red) is placed at the center of the chamber directly behind the nozzle. After the interaction, the clusters are removed by a roots pump of type Leybold RUVAC WH 7000. The motor of the cold head is located outside of the interaction chamber, while the main parts of the cluster-jet target including the cryogenic parts of the cold head extend into the chamber. A cold finger between the cold stage and the nozzle is installed to realize an interaction of the laser with the cluster beam behind the nozzle at the center of the interaction chamber. 
In order to offer the possibility to cool the target gas before entering the nozzle to temperatures of about $\mathrm{T}=60 \mathrm{~K}$, required to increase the target density as well as the cluster size, a two-stage cold head is used (cf. Table I, Figs. 2 and 3). The applied cold head works with a closed helium cycle in combination with a compressor. Both parts are connected via flexible high pressure lines. A displacer inside the cold head separates the two volumes. According to the Gifford-McMahon process, the movement of the displacer leads to a compression and expansion of the helium within the volumes resulting in a heat exchange with the compressor. The first stage of the cold head, the warm stage, pre-cools the gas, and the

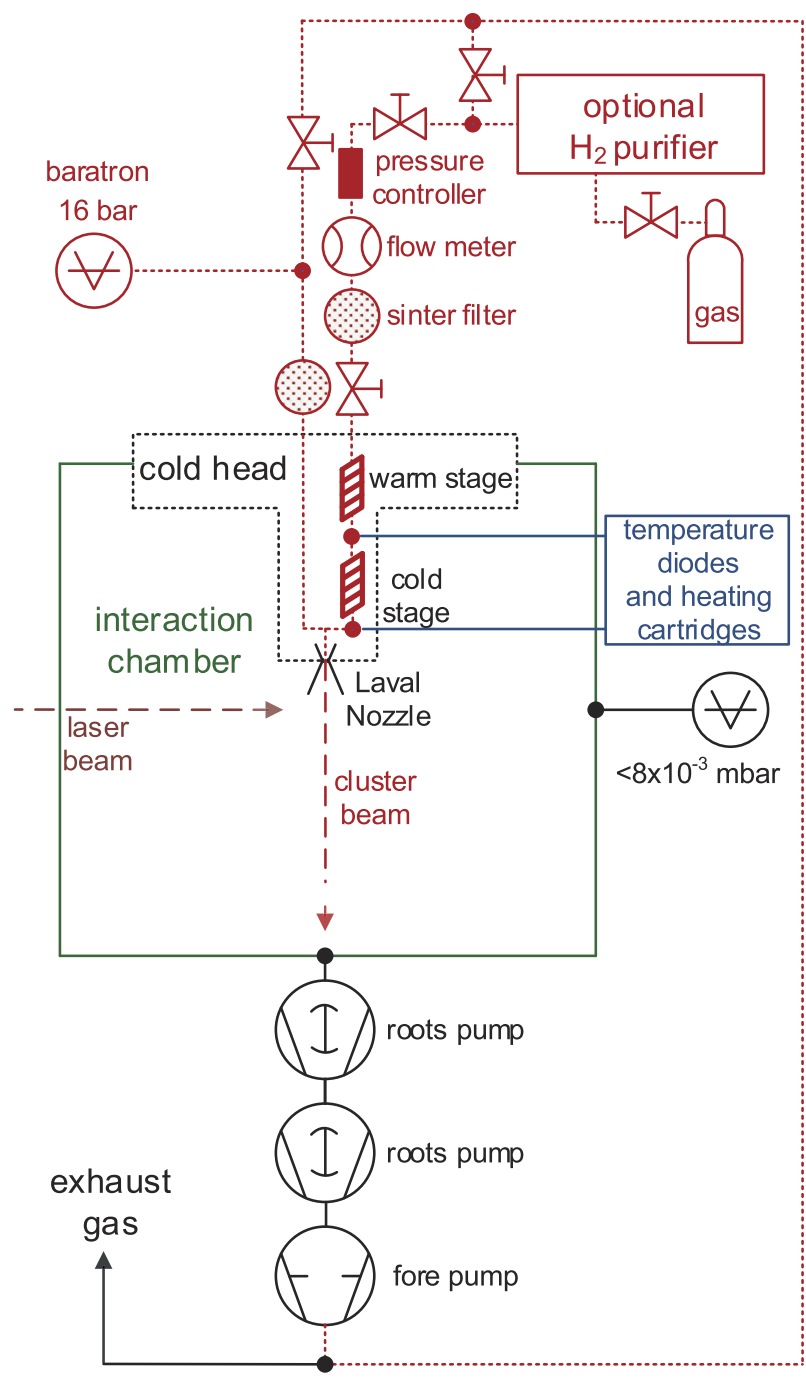

FIG. 3. Sketch of the gas (red) and vacuum system of the cluster-jet target MCT1S installed at the interaction chamber. A cold head is used to cool the target gas. A pressure controller, a flow meter, temperature diodes, and heating cartridges are used to monitor and control the target parameters. The interaction zone between the laser and the cluster beam is located directly behind the Laval nozzle. Opposite to the Laval nozzle, a powerful roots pump is installed to remove the clusters directly after the interaction to ensure a minimal gas background. second stage, the cold stage, realizes the final low temperatures of the nozzle.

Before entering the cold head, the target gas is guided through a pressure controller, to monitor and control the pressure of the gas in front of the nozzle, and is then fed to a flow meter (see Fig. 3). The information of the flow meter is used to calculate the minimum diameter of the nozzle at a given pressure which offers the possibility to recognize a blocked nozzle. Due to the very small diameter of the nozzle of, e.g., $\emptyset_{\mathrm{H}_{2}}=28 \mu \mathrm{m}$ for hydrogen, the gas is additionally purified using a palladium hydrogen purifier. Therefore, the hydrogen gas has an impurity level of better than $1 \mathrm{ppb}$ directly behind the device. The target gas is directed through pipes wrapped around the two stages of the cold head (see Fig. 2). The pipes located at the cooling parts of the cold head are made from copper to guarantee an optimal heat exchange between the cold head and the gas. The gas pipes in between the cooling parts are made from stainless steel to reduce a direct heat exchange between the cryogenic stages for an optimal cooling of the gas. Two additional sinter filters are installed to avoid a blocking of the nozzle due to possible impurities within the piping system of the target gas. Finally, the cooled gas enters a cold finger mounted directly at the top of the cold stage of the cold head. The cold finger consists of copper and is surrounded by a small walled hollow cylinder made from reflecting stainless steel acting as heat radiation shielding. At the end of the cold finger, the Laval nozzle is mounted. Both, the cold finger as well as the nozzle are sealed with a cylindrical indium sealing. This sealing is also gas tight at cryogenic temperatures and, therefore, ideally suited as nozzle sealing in the temperature range of importance here.

At the two stages of the cold head, temperature diodes and heating cartridges are installed to monitor and control the temperatures of both stages. The equipment of the cluster-jet target is remotely controlled by a slow control system realized in LabVIEW by National Instruments, ${ }^{30}$ which is also used to display the target parameters on a website and to store them every $10 \mathrm{~s}$. With this system, it could be shown that the stagnation conditions and consequently the characteristics of the cluster beam achieve a stability of better than $\Delta T=0.1 \mathrm{~K}$ and $\Delta p=0.1$ bar.

For the experiments presented here, the cluster-jet target is directly integrated in a removable interaction vacuum chamber plate and extends appropriately into the interaction chamber. For this reason, the laser pulse can be directly focused onto the cluster beam. The modular design allows for a quick disassembly and precise re-installation. The setup presented here allows for a very quick and simple change between different gases as the target material. Depending on the gas, only the nozzle has to be exchanged and this can be done without dismantling the complete target. As a result, a series of measurements with various gas types can be performed rapidly.

TABLE II. Vacuum pumps used at the cluster-laser interaction chamber and their pumping speeds.

Vacuum pump Nominal pumping speed $\left(\mathrm{m}^{3} / \mathrm{h}\right)$

Leybold SOGEVAC SV 300 240

Leybold RUVAC WSU 1001

1000

Leybold RUVAC WH 7000 
Opposite to the cluster-jet target, a powerful roots pump is installed to directly remove the clusters after the interaction with the laser beam to ensure a good vacuum in the interaction chamber (Fig. 2, Table II). With this powerful pumping system, the vacuum in the interaction chamber was below $8 \times 10^{-3}$ mbar during the measurements with a hydrogen gas flow of $0.016 \mathrm{l}_{\mathrm{n}} / \mathrm{s}$.

\section{CLUSTER-JET CHARACTERIZATION}

The cluster beam was characterized which is required for laser plasma applications. Of special interest is the target volume density at the position of the laser interaction point. Although a direct measurement, in principle, is possible, due to the experimental setup, such a measurement would be accompanied with large systematic uncertainties. Alternatively, a much more reliable method is an estimation using the gas flow through the nozzle. Since the gas flow meter information was designed for higher gas flows at lower temperatures, the gas flow is calculated based on the well known stagnation conditions and the nozzle geometry. The gas volume flow $q_{\mathrm{v}}$ through the nozzle can be calculated using

$$
q_{v}=A^{*} \frac{p_{0}}{\sqrt{M T_{0}}} \frac{T_{\mathrm{N}}}{p_{\mathrm{N}}}\left(\frac{2}{\kappa+1}\right)^{\frac{\kappa+1}{2(\kappa-1)}} \sqrt{\kappa R},
$$

where $A^{*}$ is the cross section of the narrowest point of the nozzle, $p_{0}$ and $T_{0}$ are the stagnation conditions of the gas in front of the nozzle, $p_{\mathrm{N}}=1.01325 \mathrm{bar}$ and $T_{\mathrm{N}}=273.15 \mathrm{~K}$ are the normal pressure and temperature, $M$ is the molar mass of the gas, $\kappa=C_{\mathrm{p}} / C_{\mathrm{V}}$ is the heat capacity ratio, and $R$ is the universal gas constant. A calculation of the mass flow $\dot{m}$ with respect to the gas flow $q_{v}$

$$
\dot{m}=\frac{q_{v} \cdot M \cdot p_{\mathrm{N}}}{R \cdot T_{\mathrm{N}}}
$$

allows for a determination of the target volume density $\rho_{\text {volume }}(x)$ with the cluster beam cross section $A_{\text {beam }}(x)$ at the distance $x$ to the nozzle and the Avogadro constant $N_{\mathrm{A}}$

$$
\rho_{\text {volume }}(x)=\frac{\dot{m}}{v \cdot A_{\text {beam }}(x)} \cdot \frac{N_{\mathrm{A}}}{M} .
$$

For the temperatures used, the mean cluster velocity $v$ can be approximately estimated assuming an ideal gas ${ }^{27}$

$$
v=\sqrt{\frac{2 \kappa}{\kappa-1} \frac{R T_{0}}{M}} .
$$

In the case of a real gas, the jet velocity might be lower by, e.g., a few percent. Therefore, the estimated target density might be larger by this value. Obviously, the target density depends on the gas used and the stagnation conditions, which can easily be defined just by changing the nozzle temperature and/or the gas pressure. The propagation of the cluster beam after leaving the nozzle is approximately based on the intercept theorem. ${ }^{32}$ Assuming a circular volume element of the cluster beam, the density decreases by $\sim 1 / x^{2}$ by increasing the distance $x$ from the nozzle. Taking this into account, the measurements were performed at $28 \mathrm{~mm}$ behind the nozzle exit. The estimated target densities at the interaction point are listed in Table III. The cluster beam diameter at the interaction point was calculated considering the opening angle of the nozzle of $\alpha=7^{\circ}$ as the cluster beam divergence. However, it has to be noted that previous measurements at much lower temperatures of, e.g., $25 \mathrm{~K}$ and with liquid hydrogen entering the nozzle allowed for an optical observation of the jet beam. ${ }^{32}$ These observations showed a smaller divergence in the range of $\alpha=5^{\circ}-7^{\circ 32}$ resulting in higher target densities.

In addition to the cluster beam density, the average number $N$ of atoms per cluster is an important parameter. It can be predicted using the empirical Hagena's scaling law ${ }^{33}$

$$
N=A_{N}\left(\frac{\gamma^{*}}{1000}\right)^{\gamma_{N}}
$$

where $A_{N}=33$ and $\gamma_{N}=2.35$ are empirical values for a Hagena parameter $\gamma^{*}>1800{ }^{33,34}$ The Hagena parameter $\gamma^{*}$,

$$
\gamma^{*}=\frac{\hat{k} p_{0}\left(\frac{0.74 d_{\mathrm{n}}}{\tan \alpha_{1 / 2}}\right)^{0.85}}{T_{0}^{2.29}},
$$

predicts the formation of clusters, and a value of $\gamma^{*}>1000$ leads to a pronounced formation of clusters. $\hat{k}_{\mathrm{H}_{2}}=184$ is a gas dependent constant, ${ }^{35} d_{\mathrm{n}}$ is the narrowest inner nozzle diameter, and $\alpha_{1 / 2}=3.5^{\circ}$ is the half angle of the nozzle.

The Hagena parameter and scaling law are based on measurements performed with nitrogen, carbon dioxide, and argon. In the case of hydrogen, mass distribution measurements were successfully performed at the University of Münster and resulted in a good agreement within an additional constant underestimation by a factor of 2.6. ${ }^{32}$ This correction factor for hydrogen will be considered in the following cluster size calculations. Moreover, it was shown that the cluster mass distribution can be described by a log-normal distribution

$$
f(x, \mu, \sigma)=\frac{1}{x \sigma \sqrt{2 \pi}} \exp \left(-\frac{(\ln (x)-\mu)^{2}}{2 \sigma^{2}}\right),
$$

with the scaling parameters $\mu$ and $\sigma$, and the arithmetic mean

$$
N=\exp \left(\mu+\frac{\sigma^{2}}{2}\right)
$$

TABLE III. Calculated and measured target parameters and characteristics for the parameter sets investigated. The density was estimated based on the calculated gas flow through the nozzle.

\begin{tabular}{lccccc}
\hline \hline Gas & Pressure (bar) & Temperature (K) & Density (atoms $\left./ \mathrm{cm}^{3}\right)$ & Predicted cluster diameter (nm) & Measured cluster diameter (nm) \\
\hline Hydrogen & $16.0 \pm 0.1$ & $57.0 \pm 0.1$ & $(3.4 \pm 0.2) \times 10^{16}$ & $35.3 \pm 1.1$ & $40 \pm 10$ \\
& $16.0 \pm 0.1$ & $66.0 \pm 0.1$ & $(2.9 \pm 0.2) \times 10^{16}$ & $27.1 \pm 0.8$ & $20_{-15}^{+20}$ \\
\hline \hline
\end{tabular}


The diameter of a cluster $d_{\text {Cluster }}$ can be calculated using the average number $N$ of atoms per cluster

$$
d_{\text {Cluster }}=2\left(\frac{3 m N}{4 \pi \rho_{\text {Cluster }}}\right)^{\frac{1}{3}} .
$$

The density of a cluster $\rho_{\text {Cluster }}$ is assumed to be the solid density of the gas used, and $m$ is the mass of the hydrogen atom. The estimated average cluster diameters for the measurements presented are shown and compared to measurements using Mie-scattering in Table III.

\section{CLUSTER SIZE MEASUREMENTS USING MIE-SCATTERING}

An experimental verification of the cluster size was carried out using Mie-scattering. ${ }^{36}$ The cluster size is important for the understanding of the proton-acceleration process, and additionally, it is very useful for a comparison with theoretical predictions described in Sec. III. As illustrated in Fig. 4, a $3 \mathrm{~W}$ frequency-doubled solid state laser at $532 \mathrm{~nm}$ was used which was focused to a spot size of about $50 \mu \mathrm{m}$ onto the cluster-jet $28 \mathrm{~mm}$ downstream from the Laval nozzle. In the following, the laser forward direction is defined as $0^{\circ}$. Before entering the vacuum, a $\lambda / 2$ plate was installed to change the laser polarization direction. The scattering volume was limited by a slit with a width of about $5 \mathrm{~mm}$ in the laser propagation direction to define the interaction region. The scattered light was collected by an achromatic lens with a focal length of $75 \mathrm{~mm}$ and with an acceptance angle of $23 \mathrm{mrad}$; the interaction point was imaged

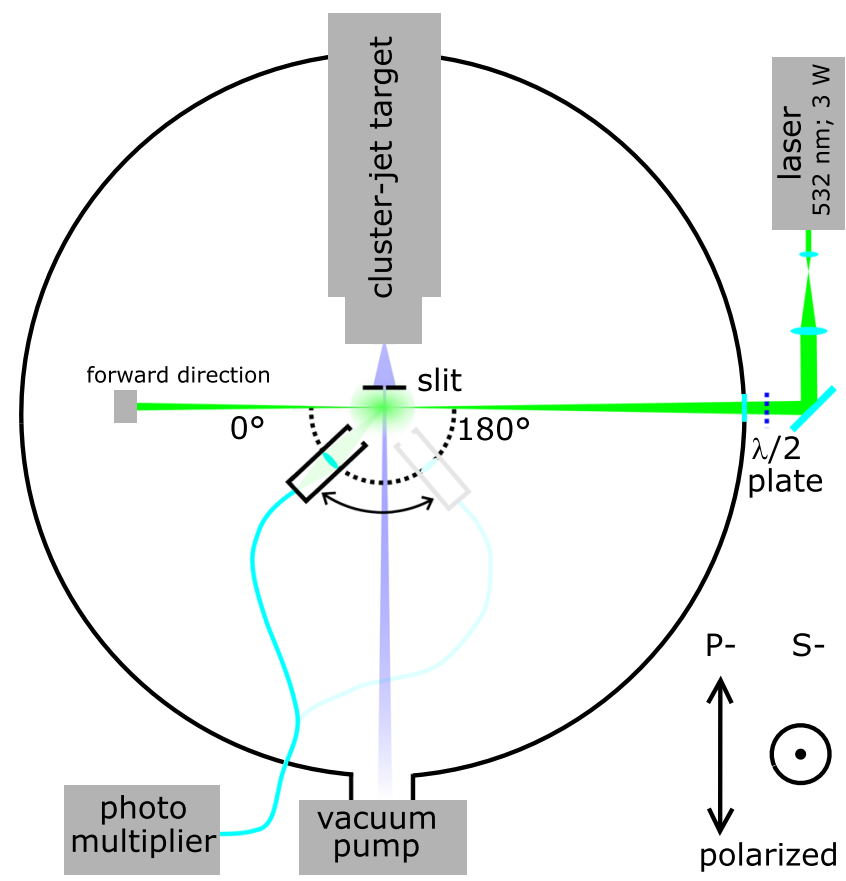

FIG. 4. Setup of the Mie-scattering measurement. The laser beam is focused into the cluster-jet, and the scattered light is collected at different angles with respect to the laser forward direction for the polarizations $\mathrm{S}$ and $\mathrm{P}$. onto a glass fiber. This imaging assembly was placed on a rotatable stage and could be rotated between $20^{\circ}$ and $130^{\circ}$ in the laser incidence plane. The fiber was coupled to a photomultiplier (PMHamamatsu R928) outside the vacuum chamber, and the relative voltage was recorded. The angularly dependent scattering of the perpendicular $(\mathrm{S})$ - or parallel $(\mathrm{P})$-polarized laser light, with respect to the detection plane, was measured. The intensity $I_{0}(\Theta, \alpha(N))$ scattered by a single cluster, composed of $N$ atoms, into an angle $\Theta$, is given by

$$
I_{0}(\Theta, \alpha(N))=\left(\frac{\lambda}{2 \pi R}\right)^{2} I_{0} i_{\perp ; \|}(\Theta, \alpha(N)),
$$

where $\alpha(N)=\frac{2 \pi a(N)}{\lambda}$ is the size parameter, which depends on the incident wavelength $\lambda$ and the Mie-radius $a . R$ is the distance between the detector and the interaction point, $I_{0}$ is the initial intensity, and $i_{\perp ; \|} \equiv i_{\perp ; \|} / \alpha^{3}$ is a fitting parameter for the S- and P-polarized case, respectively. A change of the polarization of the incident laser light results in a different scattering behavior. While perpendicular polarized light is scattered homogeneously, only having an asymmetric intensity in forward and backward directions, the parallel polarized light has an additional minimum at $90^{\circ}$ with respect to the direction of the incident light.

Since there can be more than one cluster in the scattering volume, the total intensity scattered is given by the integral over all clusters consisting of $N$ atoms in the volume

$$
I(\Theta)=V_{0} \int_{0}^{\infty} I_{0}(\Theta, \alpha(N)) f(N) \mathrm{d} N
$$

Here, $f(N)$ is the particle size distribution depending, on the one hand, on the spatial position within the cluster jet and on the other hand, defined by the log-normal distribution of the statistical

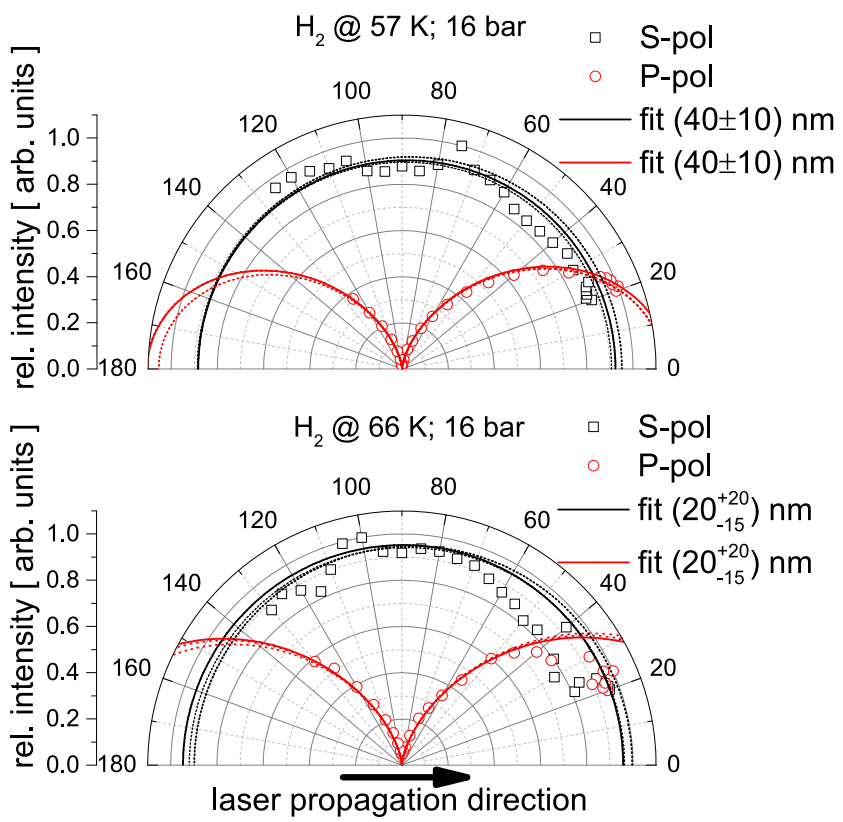

FIG. 5. Measurement of the different cluster size diameters: $\mathrm{H}_{2}$ at $57 \mathrm{~K}: d=(40 \pm$ 10) $\mathrm{nm}$ and $\mathrm{H}_{2}$ at $66 \mathrm{~K}: d=\left(20_{-15}^{+20}\right) \mathrm{nm}$. 
generation process of the clusters. The insertion of the slit $5 \mathrm{~mm}$ in width directly behind the cluster nozzle leads to a decrease in the spatial distribution of the cluster-jet beam resulting in a well defined scattering volume. However, although in principle possible, due to the limited covered scattering angle range and angular resolution, this setup does not allow us to resolve the log-normal particle size distribution. Based on that, the assumption was made that the measured values are a reflection of the average size of the clusters. Following the theoretical approach described by Bohren, ${ }^{3}$ the theoretical Mie-scattering distributions for P- and S-polarized light were calculated and fitted to the measured values. Note that in the presented case, the use of different polarizations instead of different wavelengths results ${ }^{23}$ in a verification of the measurements. For the calculated values of $i_{\perp ; \|}$, the refractive index depending on the laser wavelength $\lambda$ needs to be taken into account. For hydrogen, $n_{\mathrm{H}_{2}}$; liq. $=1.117^{38}$ was assumed. Figure 5 illustrates the measured values (symbols) and the theoretical fitted diameters (lines). The dashed lines represent the corresponding error range. A smaller scattering source results in a more spatially isotropic scatter behavior. For this reason, the errors become large in this case. The results are summarized in Table III. The predicted cluster diameters using the Hagena formula and the measured cluster diameters via the Mie-scattering setup are in good agreement within the uncertainties.

\section{RESULTS OF LASER-DRIVEN ACCELERATION USING THE HYDROGEN CLUSTER-JET TARGET}

Preliminary measurements of proton acceleration using the hydrogen cluster-jet target at the ARCTURUS laser facility of the University of Düsseldorf were performed. A Ti:Sapphire laser with an energy of up to $7 \mathrm{~J}$ before compression, a pulse duration of $30 \mathrm{fs}$, and a beam diameter of $100 \mathrm{~mm}$ was used to irradiate the clusterjet beam. For a first benchmark test, a fraction of this energy of about $700 \mathrm{~mJ}$ was focused onto the cluster beam by an $\mathrm{f} / 2$ off-axis parabola to a focal diameter of $4 \mu \mathrm{m}$ (FWHM) resulting in an intensity of about $10^{20} \mathrm{~W} / \mathrm{cm}^{2}$. For the results reported here, the hydrogen gas in front of the Laval nozzle had a temperature of roughly $50 \mathrm{~K}$ and a pressure of 16 bar resulting in a cluster size of roughly $d=45 \mathrm{~nm}$.

The proton spectrum was measured along the laser forward direction by a Thomson-parabola spectrometer. ${ }^{39}$ Figure 6(a) shows the raw image on the detector demonstrating the proton track. In Fig. 6(b), the energy spectrum is presented which was extracted employing a MATLAB routine. In this measurement, maximum energies of roughly $60 \mathrm{keV}$ were observed. From more extended studies, which will be presented elsewhere, a high shot-to-shot stability of the accelerated protons can be deduced. With a laser energy stability of about $3 \%$, a proton cutoff-energy of about $(60 \pm 6) \mathrm{keV}$ for several 10s of shots was obtained. This $10 \%$-stability is achieved as the driving mechanism is the Coulomb-explosion process. ${ }^{40}$ Coulomb-explosion is for these small clusters very robust to laser parameter fluctuations since it depends only on the cluster size and not so much on the given laser intensity.

The calculated cluster target density in combination with the average number of atoms within one cluster predicted via the Hagena formula allows for the estimation of the number of clusters within the focal volume $\left(\mathrm{V}_{\mathrm{Foc}}=189 \mu \mathrm{m}^{3}\right)$, defined as the volume

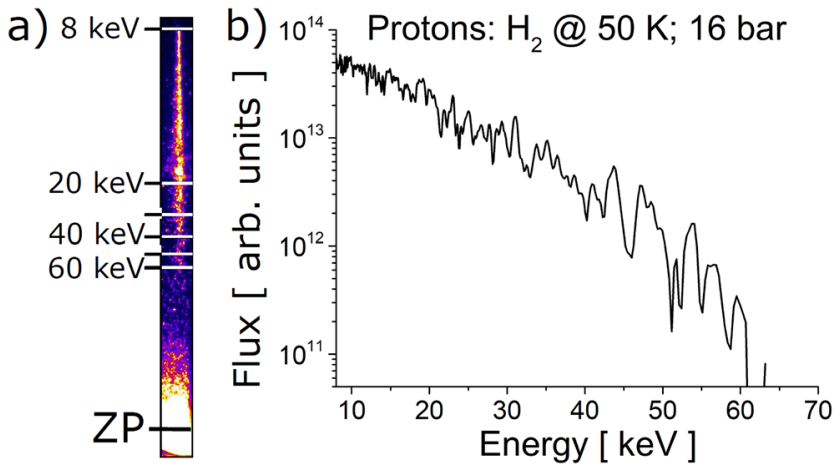

FIG. 6. (a) Raw data image of a Thomson-parabola spectrometer. (b) Evaluated energy spectrum with a cut-off energy of roughly $60 \mathrm{keV}$.

within one Rayleigh-length of the laser focus. In the case of the proton-acceleration measurement, the mean cluster number in the focal volume was determined to be 13 . Consequently there is more than one single cluster located in the interaction volume which forms the basis for stable and reproducible proton spectra.

\section{CONCLUSION}

A cluster-jet target was designed, built, and successfully commissioned at the University of Münster. This target can be used with different gases and allows for a stable and continuous flow of the pure target material. Additionally, this cluster-jet target was implemented at the ARCTURUS laser facility of the University of Düsseldorf for laser-driven proton acceleration. A detailed technical description of the cluster source demonstrates the characteristics of such a target. Theoretical calculations of the essential cluster parameters, like density and cluster size, using the Hagena relation for different stagnation conditions were performed. Moreover, the diameters of hydrogen clusters were measured using a Miescattering technique and the results demonstrate a good agreement with the Hagena prediction within the errors. Preliminary measurements using hydrogen clusters irradiated by a high-intensity $5 \mathrm{~Hz}$ laser prove the operational reliability of the target system by observing protons in the multi-kiloelectron volt range. Based on these results, a newly designed cluster-jet target is currently under construction at the University of Münster. This target will extend the region of accessible stagnation conditions even toward the generation of hydrogen clusters from the liquid phase, which will result in significantly larger cluster sizes and higher densities allowing considerable higher kinetic energies of the accelerated protons to be achieved.

\section{ACKNOWLEDGMENTS}

We acknowledge financial support by the German Federal Ministry of Education and Research (BMBF) joint research Project Nos. 05K16PM3 and 05K16PFA. The work provided by the teams of the mechanical and electronic workshops at Münster University is very much appreciated, and we thank them for the excellent manufacturing of the various components. We thank A. Alejo from Queens University Belfast for the spectrometer evaluation routine. 


\section{REFERENCES}

${ }^{1}$ S. C. Wilks, A. B. Langdon, T. E. Cowan, M. Roth, M. Singh, S. Hatchett, M. H. Key, D. Pennington, A. MacKinnon, and R. A. Snavely, "Energetic proton generation in ultra-intense laser-solid interactions," Phys. Plasmas 8, 542-549 (2001).

${ }^{2}$ P. Mora, "Plasma expansion into a vacuum," Phys. Rev. Lett. 90, 185002 (2003)

${ }^{3}$ A. Henig, S. Steinke, M. Schnurer, T. Sokollik, R. Horlein, D. Kiefer, D. Jung, J. Schreiber, B. M. Hegelich, X. Q. Yan, J. Meyer-ter Vehn, T. Tajima, P. V. Nickles, W. Sandner, and D. Habs, "Radiation-pressure acceleration of ion beams driven by circularly polarized laser pulses," Phys. Rev. Lett. 103, 245003 (2009).

${ }^{4}$ X. Q. Yan, C. Lin, Z. M. Sheng, Z. Y. Guo, B. C. Liu, Y. R. Lu, J. X. Fang, and J. E. Chen, "Generating high-current monoenergetic proton beams by a circularly polarized laser pulse in the phase-stable acceleration regime," Phys. Rev. Lett. 100, 135003 (2008)

${ }^{5}$ B. Aurand, S. Kuschel, O. Jaeckel, C. Roedel, H. Y. Zhao, S. Herzer, A. E. Paz, J. Bierbach, J. Polz, B. Elkin, G. G. Paulus, A. Karmakar, P. Gibbon, T. Kuehl, and M. C. Kaluza, "Radiation pressure-assisted acceleration of ions using multicomponent foils in high-intensity laser-matter interactions," New J. Phys. 15, 033031 (2013)

${ }^{6}$ L. O. Silva, M. Marti, J. R. Davies, R. A. Fonseca, C. Ren, F. S. Tsung, and W. B. Mori, "Proton shock acceleration in laser-plasma interactions," Phys. Rev. Lett. 92, 015002 (2004).

${ }^{7}$ F. Fiuza, A. Stockem, E. Boella, R. A. Fonseca, L. O. Silva, D. Haberberger, S. Tochitsky, C. Gong, W. B. Mori, and C. Joshi, "Laser-driven shock acceleration of monoenergetic ion beams," Phys. Rev. Lett. 109, 215001 (2012).

${ }^{8}$ L. Yin, B. J. Albright, B. M. Hegelich, and J. C. Fernandez, "GeV laser ion acceleration from ultrathin targets: The laser break-out afterburner," Laser Part. Beams 24, 291-298 (2006).

${ }^{9}$ D. Jung, L. Yin, D. C. Gautier, H. C. Wu, S. Letzring, B. Dromey, R. Shah, S. Palaniyappan, T. Shimada, R. P. Johnson, J. Schreiber, D. Habs, J. C. Fernandez, B. M. Hegelich, and B. J. Albright, "Laser-driven $1 \mathrm{GeV}$ carbon ions from preheated diamond targets in the break-out afterburner regime," Phys. Plasmas 20, 083103 (2013).

${ }^{10}$ B. Aurand, B. Elkin, L. O. Heim, B. Lommel, B. Kindler, M. Tomut, C. Roedel, S. Kuschel, O. Jaeckel, J. Barz, and T. Kuehl, "Preparation and characterization of nanometer-thin freestanding polymer foils for laser-ion acceleration," J. Polym. Sci., Part B: Polym. Phys. 51, 1355-1360 (2013).

${ }^{11}$ B. Aurand, B. Elkin, L. O. Heim, B. Lommel, B. Kindler, M. Tomut, C. Roedel, S. Kuschel, O. Jaeckel, and T. Kuehl, "Ultra-thin polymer foils for laser-ion acceleration," J. Radioanal. Nucl. Chem. 299, 965-968 (2014).

${ }^{12}$ A. Macchi, M. Borghesi, and M. Passoni, "Ion acceleration by superintense laser-plasma interaction," Rev. Mod. Phys. 85, 751-793 (2013).

${ }^{13} \mathrm{~A}$. Macchi, "A review of laser-plasma ion acceleration," preprint arXiv:1712.06443 (2017).

${ }^{14}$ S. Buffechoux, J. Psikal, M. Nakatsutsumi, L. Romagnani, A. Andreev, K. Zeil, M. Amin, P. Antici, T. Burris-Mog, A. Compant-La-Fontaine, E. d'Humieres, S. Fourmaux, S. Gaillard, F. Gobet, F. Hannachi, S. Kraft, A. Mancic, C. Plaisir, G. Sarri, M. Tarisien, T. Toncian, U. Schramm, M. Tampo, P. Audebert, O. Willi, T. E. Cowan, H. Pepin, V. Tikhonchuk, M. Borghesi, and J. Fuchs, "Hot electrons transverse refluxing in ultraintense laser-solid interactions," Phys. Rev. Lett. 105, 015005 (2010).

${ }^{15}$ O. Tresca, D. C. Carroll, X. H. Yuan, B. Aurand, V. Bagnoud, C. M. Brenner, M. Coury, J. Fils, R. J. Gray, T. Kuhl, C. Li, Y. T. Li, X. X. Lin, M. N. Quinn, R. G. Evans, B. Zielbauer, M. Roth, D. Neely, and P. McKenna, "Controlling the properties of ultraintense laser-proton sources using transverse refluxing of hot electrons in shaped mass-limited targets," Plasma Phys. Controlled Fusion 53, 105008 (2011).

${ }^{16}$ I. Prencipe, J. Fuchs, S. Pascarelli, D. W. Schumacher, R. B. Stephens, N. B. Alexander, R. Briggs, M. Büscher, M. O. Cernaianu, A. Choukourov, M. De Marco, A. Erbe, J. Fassbender, G. Fiquet, P. Fitzsimmons, C. Gheorghiu, J. Hund, L. G. Huang, M. Harmand, N. J. Hartley, A. Irman, T. Kluge, Z. Konopkova, S. Kraft, D. Kraus, V. Leca, D. Margarone, J. Metzkes, K. Nagai, W. Nazarov, P. Lutoslawski, D. Papp, M. Passoni, A. Pelka, J. P. Perin, J. Schulz, M. Smid, C. Spindloe, S. Steinke, R. Torchio, C. Vass, T. Wiste, R. Zaffino, K. Zeil, T. Tschentscher, U. Schramm, and T. E. Cowan, "Targets for high repetition rate laser facilities: Needs, challenges and perspectives," High Power Laser Sci. Eng. 5 e17 (2017).

${ }^{17}$ M. H. Helle, D. F. Gordon, D. Kaganovich, Y. Chen, J. P. Palastro, and A. Ting, "Laser-accelerated ions from a shock-compressed gas foil," Phys. Rev. Lett. 117, 165001 (2016).

${ }^{18}$ L. Willingale, S. P. Mangles, P. M. Nilson, R. J. Clarke, A. E. Dangor, M. C. Kaluza, S. Karsch, K. L. Lancaster, W. B. Mori, Z. Najmudin, J. Schreiber, A. G. Thomas, M. S. Wei, and K. Krushelnick, "Collimated multi-Mev ion beams from high-intensity laser interactions with underdense plasma," Phys. Rev. Lett. 96, 245002 (2006).

${ }^{19}$ S. N. Chen, M. Vranic, T. Gangolf, E. Boella, P. Antici, M. Bailly-Grandvaux, P. Loiseau, H. Pepin, G. Revet, J. J. Santos, A. M. Schroer, M. Starodubtsev, O. Willi, L. O. Silva, E. d'Humieres, and J. Fuchs, "Collimated protons accelerated from an overdense gas jet irradiated by a $1 \mu \mathrm{m}$ wavelength high-intensity short-pulse laser," Sci. Rep. 7, 13505 (2017).

${ }^{20}$ S. Karsch, S. Dusterer, H. Schwoerer, F. Ewald, D. Habs, M. Hegelich, G. Pretzler, A. Pukhov, K. Witte, and R. Sauerbrey, "High-intensity laser induced ion acceleration from heavy-water droplets," Phys. Rev. Lett. 91, 015001 (2003).

${ }^{21}$ M. Schnurer, S. Ter-Avetisyan, S. Busch, E. Risse, M. P. Kalachnikov, W. Sandner, and P. Nickles, "Ion acceleration with ultrafast laser driven water droplets," Laser Part. Beams 23, 337-343 (2005).

${ }^{22}$ P. Hilz, T. M. Ostermayr, A. Huebl, V. Bagnoud, B. Borm, M. Bussmann, M. Gallei, J. Gebhard, D. Haffa, J. Hartmann, T. Kluge, F. H. Lindner, P. Neumayr, C. G. Schaefer, U. Schramm, P. G. Thirolf, T. F. Rosch, F. Wagner, B. Zielbauer, and J. Schreiber, "Isolated proton bunch acceleration by a petawatt laser pulse," Nat. Commun. 9, 423 (2018).

${ }^{23}$ R. Prasad, M. Borghesi, F. Abicht, P. V. Nickles, H. Stiel, M. Schnurer, and S. Ter-Avetisyan, "Ethanol $\left(\mathrm{C}_{2} \mathrm{H}_{5} \mathrm{OH}\right)$ spray of sub-micron droplets for laser driven negative ion source," Rev. Sci. Instrum. 83, 083301 (2012).

${ }^{24}$ Y. Fukuda, A. Y. Faenov, M. Tampo, T. A. Pikuz, T. Nakamura, M. Kando, Y. Hayashi, A. Yogo, H. Sakaki, T. Kameshima, A. S. Pirozhkov, K. Ogura, M. Mori, T. Esirkepov, J. Koga, A. S. Boldarev, V. A. Gasilov, A. I. Magunov, T. Yamauchi, R. Kodama, P. R. Bolton, Y. Kato, T. Tajima, H. Daido, and S. V. Bulanov, "Energy increase in multi-MeV ion acceleration in the interaction of a short pulse laser with a cluster-gas target," Phys. Rev. Lett. 103, 165002 (2009).

${ }^{25}$ S. Jinno, H. Tanaka, R. Matsui, M. Kanasaki, H. Sakaki, M. Kando, K. Kondo, A. Sugiyama, M. Uesaka, Y. Kishimoto, and Y. Fukuda, "Characterization of micron-size hydrogen clusters using mie scattering," Opt. Express 25, 18774-18783 (2017).

${ }^{26}$ S. Jinno, M. Kanasaki, M. Uno, R. Matsui, M. Uesaka, Y. Kishimoto, and Y. Fukuda, "Micron-size hydrogen cluster target for laser-driven proton acceleration," Plasma Phys. Controlled Fusion 60, 044021 (2018).

${ }^{27}$ A. Täschner, "Entwicklung und Untersuchung von Cluster-Jet-Targets höchster Dichte," Ph.D. thesis, Westfälische Wilhelms-Universität Münster, Germany, 2012.

${ }^{28}$ S. Grieser, "Das Cluster-Jet Target MCT1S und die Analyse von Clusterstrahlen," M.S. thesis, Westfälische Wilhelms-Universität Münster, Germany, 2014.

${ }^{29} \mathrm{~S}$. Grieser, "Cluster-jet targets for the PANDA-, MAGIX-, and CryoFlashexperiments at hadron-, lepton-, and laser-facilities," Ph.D. thesis, Westfälische Wilhelms-Universität Münster, 2018.

${ }^{30}$ See http://www.ni.com/de-de.html for National Instruments, 2018.

${ }^{31}$ M. Wutz, H. Adam, and H. Walcher, Theorie und Praxis der Vakuumtechnik, 4th ed. (Friedrich Vieweg \& Sohn, 1988)

${ }^{32}$ E. Köhler, "Mass spectroscopy of hydrogen cluster-jets and beam density optimisation studies," Ph.D. thesis, Westfälische Wilhelms-Universität Münster, Germany, 2015

${ }^{33}$ O. F. Hagena, “Cluster ion sources (invited)," Rev. Sci. Instrum. 63, 2374-2379 (1992).

${ }^{34}$ O. Hagena, "Condensation in free jets: Comparison of rare gases and metals," Z. Phys. D At., Mol. Clusters 4, 291-299 (1987).

${ }^{35}$ R. A. Smith, T. Ditmire, and J. W. G. Tisch, "Characterization of a cryogenically cooled high-pressure gas jet for laser/cluster interaction experiments," Rev. Sci. Instrum. 69, 3798-3804 (1998). 
${ }^{36}$ G. Mie, "Beiträge zur Optik trüber Medien, speziell kolloidaler Metallösungen," Ann. Phys. 330, 377-445 (1908).

${ }^{37}$ C. F. Bohren and D. R. Huffman, Absorption and Scattering of Light by Small Particles (Wiley VCH GmbH \& Co. KgaA, 1998).

${ }^{38} \mathrm{H}$. E. Johns and J. O. Wilhelm, "The refractive indices of liquid oxygen, nitrogen, and hydrogen," Can. J. Res. 15a, 101-108 (1937).

${ }^{39}$ D. Gwynne, S. Kar, D. Doria, H. Ahmed, M. Cerchez, J. Fernandez, R. J. Gray, J. S. Green, F. Hanton, D. A. MacLellan, P. McKenna, Z. Najmudin,
D. Neely, J. A. Ruiz, A. Schiavi, M. Streeter, M. Swantusch, O. Willi, M. Zepf, and M. Borghesi, "Modified thomson spectrometer design for high energy, multi-species ion sources," Rev. Sci. Instrum. 85, 033304 (2014).

${ }^{40}$ K. Nishihara, H. Amitani, M. Murakami, S. Bulanov, and T. Esirkepov, "High energy ions generated by laser driven coulomb explosion of cluster," Nucl. Instrum. Methods Phys. Res., Sect. A 464, 98-102 (2001). 